\title{
Reduced RKIP expression levels are associated with frequent non-small cell lung cancer metastasis and STAT3 phosphorylation and activation
}

\author{
ANSHENG WANG ${ }^{1,2}$, GUIXIN DUAN ${ }^{2}$, CHENGLING ZHAO $^{3}$, YUAN GAO $^{2}$, \\ XUEGANG LIU ${ }^{4}$, ZUYI WANG ${ }^{2}$, WEI $\mathrm{LI}^{3}, \mathrm{KANGWU} W A N G^{2}$ and WEI WANG ${ }^{2}$
}

${ }^{1}$ Shandong University School of Medicine, Jinan, Shandong 250100; Departments of ${ }^{2}$ Thoracic Surgery, ${ }^{3}$ Respiratory Diseases and ${ }^{4}$ Cardiac Surgery, The First Affiliated Hospital of Bengbu Medical College, Bengbu, Anhui 233000, P.R. China

Received February 23, 2016; Accepted December 2, 2016

DOI: $10.3892 / 01.2017 .5846$

\begin{abstract}
The current study examined the role of Raf kinase inhibitor protein (RKIP) in non-small cell lung cancer (NSCLC) metastasis. A total of 100 patients with NSCLC were recruited following pathological diagnosis in the First Affiliated Hospital of Bengbu Medical College. The patients were classified and statistically analyzed according to their clinicopathological characteristics and tumor-node-metastasis stage. Paired tumor tissue and adjacent non-tumor tissue samples were subject to pathological diagnosis and western blot analysis. Transient transfection and lentivirus particle vector-mediated RKIP overexpression, small interfering RNA-mediated silencing, Transwell assays and immunocytochemistry methods were employed to elucidate the role and underlying mechanisms of RKIP and the Janus kinase/signal transducer and activator of transcription (JAK/STAT) signaling pathway in NSCLC metastasis. Furthermore, in order to examine the in vivo effects of RKIP, recombinant lentivirus particles containing the RKIP gene were administrated in a mouse NSCLC tumor model via tail vein injection. The results revealed reduced RKIP expression levels in NSCLC tissue compared with corresponding non-cancer tissue. Additionally, RKIP expression levels were inversely associated with NSCLC intra-lung, lymph node and long-distance metastasis. The results also indicated that RKIP was able to block STAT3 activation via phosphorylation and inhibit NSCLC-cell metastasis in vitro. Furthermore, RKIP
\end{abstract}

Correspondence to: Dr Guixin Duan, Department of Thoracic Surgery, The First Affiliated Hospital of Bengbu Medical College, 287 Huaihe River Road, Bengbu, Anhui 233000, P.R. China E-mail: guixin.duan@foxmail.com

Dr Chengling Zhao, Department of Respiratory Diseases, The First Affiliated Hospital of Bengbu Medical College, 287 Huaihe River Road, Bengbu, Anhui 233000, P.R. China

E-mail: zclbbmc@163.com

Key words: Raf kinase inhibitor protein, signal transducer and activator of transcription 3, metastasis, non-small cell lung cancer knockdown was able to promote STAT3 phosphorylation and cell metastasis in NSCLC cell lines. During in vivo experiments, RKIP overexpression was able to suppress xenograft tumor metastasis in nude mice. Therefore, RKIP may be an important factor in cancer cell metastasis in patients with NSCLC, and RKIP may inhibit NSCLC-cell invasion by blocking the activation of the JAK/STAT3 signaling pathway.

\section{Introduction}

Lung cancer has increased in incidence in the developing world since 2008, from 1.8 to 2 million per year, and this trend is predicted to continue in the future (1). Non-small cell lung cancer (NSCLC) is among the most malignant types of cancer, and has a high mortality rate (2). Approximately $80-90 \%$ of lung cancer cases are of NSCLC, and 50-70\% of these patients are diagnosed at the advanced stage of disease $(1,3)$. The survival time of patients with lung cancer is 5 years in $10-15 \%$ of cases, with the remaining exhibiting poorer survival times (1). Therefore, the early prevention of NSCLC metastasis is a key factor in lung cancer prevention and the development of novel therapeutic agents. A number of studies have investigated the mechanisms underlying NSCLC metastasis $(4,5)$; however, the exact mechanisms underlying this process require further elucidation.

Raf kinase inhibitor protein (RKIP) is a small evolutionarily conserved protein, which was initially identified to function as a physiological inhibitor of the Raf/mitogen-activated protein kinase kinase (MEK)/extracellular signal-regulated kinase (ERK) signaling pathway (6). RKIP inhibits the interaction between RAF1 and MEK, thereby preventing RAF-mediated MEK phosphorylation, which is required for signal propagation (7). RKIP is widely expressed in normal human tissues, indicating that it may function in a variety of physiological processes (8). Previous studies have suggested that RKIP may be a suppressor of cancer metastasis, as its loss of or reduced expression levels are highly associated with cancer malignancy and aggressiveness $(9,10)$. Overexpression of RKIP protein has a limited impact on the proliferation of prostate cancer cell proliferation and angiogenesis in mouse models; however, cancer cell invasion and metastasis is significantly 
reduced upon RKIP overexpression (11), indicating its potential tumor suppressor role in cancer metastasis. In a previous study, RKIP mRNA expression levels were demonstrated to be significantly downregulated in NSCLC, and lower mRNA levels were correlated with poorer differentiation and advanced tumor-node-metastasis stage in patients with NSCLC (12). However, the clinical significance of RKIP and its associations with metastasis in NSCLC, as well as its downstream mechanisms and targets, remain to be established.

Signal transducer and activator of transcription 3 (STAT3) is activated by various growth factors, cytokines and oncogenic proteins, as well as being constitutively activated in various types of malignancy (13). A previous study reported that STAT3 functions downstream of the Raf/ERK signaling pathway, and is associated with cancer metastasis (14). However, whether RKIP participates in the phosphorylation and activation of STAT3 during NSCLC cell metastasis remains to be established. A previous study reported that increased RKIP level and Raf/ERK/STAT3 pathway activation were found in metastatic NSCLC patients (15). The current study examined the role of RKIP and STAT3 phosphorylation in NSCLC, based on clinical sample analysis and in vitro and in vivo experiments.

\section{Materials and methods}

Cell lines and reagents. The A549 and NCI-H1299 human NSCLC cell lines were purchased from the American Type Culture Collection (Manassas, VA, USA). Cells were maintained in Dulbecco's modified Eagle's medium (DMEM; Thermo Fisher Scientific, Inc., Waltham, MA, USA), supplemented with $10 \%$ fetal bovine serum (Gibico; Thermo Fisher Scientific, Inc.), $100 \mathrm{U} / \mathrm{ml}$ penicillin and $100 \mu \mathrm{g} / \mathrm{ml}$ streptomycin (Sigma-Aldrich; Merck Millipore, Darmstadt, Germany) at $37^{\circ} \mathrm{C}$ in a humidified atmosphere containing 5\% $\mathrm{CO}_{2}$. Antibodies against RKIP (cat. no. 13006), STAT3 (cat. no. 9139), phosphorylated (p)STAT3 (cat. no. 9145), ERK (cat. no. 9102) and pERK (cat. no. 4370) were purchased from Cell Signaling Technology, Inc. (Danvers, MA, USA) and $\beta$-actin (cat. no. 7210) antibody was purchased from Santa Cruz Biotechnology, Inc. (Dallas, TX, USA).

Patient tissue samples and pathological diagnosis. Between March 2015 and August 2015, 100 patients with NSCLC were recruited for the present study at The First Affiliated Hospital of Bengbu Medical College (Bengbu, China). All patients underwent curative surgical resection and none had received preoperative chemotherapy or radiotherapy. All patients were diagnosed by pathological methods. The current study was performed with the approval of the Bengbu Medical College Ethics Committee, and informed consent was obtained from all patients that were recruited.

Immunohistochemical staining. Immunohistochemical staining for RKIP and phosphorylated (p)STAT3 was performed according to previous protocol (16). Briefly, antigen retrieval was performed by boiling tissue samples in an autoclave for $20 \mathrm{~min}$ in $1 \mathrm{X}$ antigen retrieval Citra solution (BioGenex, Fremont, CA, USA). The tissue samples were fixed with $4 \%$ formaldehyde at room temperature for $24 \mathrm{~h}$. After fixation, samples were sectioned at a thickness of $5 \mu \mathrm{m}$ for further analysis. Sections were blocked with PBS containing 5\% goat serum (Sigma-Aldrich; Merck Millipore) and 0.1\% Triton X-100, followed by incubation with anti-RKIP antibody (dilution, 1:200) or pSTAT3 antibody (dilution, 1:500) at $4^{\circ} \mathrm{C}$ in a humidified chamber for $12 \mathrm{~h}$. Following washes with PBS, the tissue samples were incubated at room temperature with horseradish peroxidase (HRP)-labeled secondary antibody (cat. no. 8114, 1:1,000 dilution; Cell Signaling Technology, Inc.) for $2 \mathrm{~h}$ and visualized with a 3,3'-diaminobenzidine kit (Vector Laboratories, Inc., Burlingame, CA, USA). The sections were observed and recorded using a Leica DM3000 microscope.

Western blotting. siRNA transfected cells were lysed in ice-cold lysis buffer (Bio-Rad Laboratories, Inc., Hercules, CA, USA) for $30 \mathrm{~min}$, supplemented with protease and phosphatase inhibitors (Sigma-Aldrich; Merck Millipore). Following centrifugation for $15 \mathrm{~min}$ at $12,000 \mathrm{x} g$ at $4^{\circ} \mathrm{C}$, the supernatant was collected and the protein concentration was determined using a protein assay kit (Bio-Rad Laboratories, Inc.). Total protein $(50 \mu \mathrm{g} / \mathrm{lane})$ was separated by $10 \%$ SDS-PAGE and transferred to Hybond-C nitrocellulose membranes (GE Healthcare Life Sciences, Chalfont, UK). The membranes were incubated with primary antibodies RKIP, pSTAT3 or $\beta$-actin $(1: 2,000)$ overnight at $4^{\circ} \mathrm{C}$, respectively, and subsequently probed with HRP-conjugated secondary antibodies (cat. no. 8114; 1:2,500 dilution; Cell Signaling Technology, Inc.) for $1 \mathrm{~h}$ at room temperature. Chemiluminescent signals were developed using LumiGLO ${ }^{\circledR}$ reagent (Cell Signaling Technology, Inc.) and detected using the ChemiDoc ${ }^{\mathrm{TM}}$ XRS+ gel documentation system (Bio-Rad Laboratories, Inc.). In grayscale quantification, Photoshop version 8.0 (Adobe Systems, Inc., San Jose, CA, USA) was used, setting actin grayscale as control.

Small interfering (si)RNA interference. The experiment was performed according to previous protocol (17). Briefly, $3 \times 10^{5}$ A549 and NCI-H1299 cells/2 ml were seeded in 6-well plates 1 day prior to transfection. $10 \mu \mathrm{M}$ siRNAs (cat. no. sc-36430; Santa Cruz Biotechnology, Inc.) were transfected into the cells with the aid of FuGENE ${ }^{\circledR}$ transfection reagent (Promega Corporation, Madison, WI, USA). Three days later, cells were collected using lysis assay buffer, and incubated on ice for 30 min with protease inhibitors cocktail (Sigma-Aldrich; Merck Millipore).

Transwell assay. The migration of NCI-H1299 cells in vitro was determined using Transwell assays (pore size, $8-\mu \mathrm{m}$; Corning Incorporated, Corning, NY, USA). Briefly, cell-free DMEM $(0.8 \mathrm{ml})$ with or without human interleukin-6 $(10 \mathrm{ng} / \mathrm{ml}$; BD Biosciences, San Jose, CA, USA) was placed in the lower chamber. Here, IL-6 was used as chemoattractant to induce cells migration form upper chamber to the lower plate. Microglial suspension $\left(0.1 \mathrm{ml} ; 5 \times 10^{4}\right.$ cells/well) was placed in the upper chamber and the plates were incubated for $24 \mathrm{~h}$ at $37^{\circ} \mathrm{C}$. The inserts were subsequently removed and the cells on the upper surface were removed with cotton pads. Cells on the lower surface were air dried at room temperature and stained with hematoxylin and eosin (H\&E) method after 15 min fixation at room temperature. Migration ability was quantified and 
compared by counting the number of migrated cells in the lower chamber. Five random fields at x40 magnification were counted for each condition under a phase-contrast microscope. Each experiment was repeated three times. Results were presented as the number of cells counted per field.

Lentivirus construction and infection. Lentivirus particles containing the RKIP gene were constructed using the pLenti7.3/V5-DEST ${ }^{\mathrm{TM}}$ Gateway ${ }^{\circledR}$ Vector kit (Thermo Fisher Scientific, Inc.) according to the manufacturer's protocol. The lentiviral vectors were co-transfected with virus packing vectors to form mature lentivirus particles in 293FT cells (Thermo Fisher Scientific, Inc.). The viral supernatant was harvested to determine the viral titer as previously described (18), and added to the A549 and NCI-H1299 cells for target gene expression.

Xenograft metastatic in vivo study. The current animal experiment was approved by the Bengbu Medical College Ethic Committee (Bengbu, China) in 2015. A total of 15 nude mice were randomly divided into 2 groups. Nude mice (6 weeks old, 11 male and 4 female, weight 15-18 g) were purchased from the Animal Center of Bengbu Medical College (Bengbu, China) and kept in the Animal Center for SPF level feeding at $25^{\circ} \mathrm{C}$, with a $12 \mathrm{~h}$ day/night cycle. Group one (six mice) received parenteral injection via tail vein with A549 cells as controls. Group two (nine mice) were injected with pLenti7.3-RKIP infected and overexpressed stable cells, with $\geq 90 \%$ positively expressing RKIP, using a modified tail vein injection transplantation model from a previous study (19). The transduction efficiency was determined by green fluorescent protein-positive counting under a fluorescent microscope. Briefly, 5- to 6-week-old BALB/c nude mice were injected with $1 \times 10^{6}$ A549 cells per mouse through the tail vein. At six weeks following injection, mice were examined using Xenogen IVIS imaging (PerkinElmer, Inc., Waltham, MA, USA) to detect tumor metastasis status. Subsequently, the mice were sacrificed using the cervical dislocation method. Lung tissue was collected for analysis and the tumor number and volume within the lung was measured. One part of tumor tissue was fixed for H\&E staining analysis, and the remaining was stored in a liquid nitrogen tank for subsequent studies.

Statistical analysis. Statistical analysis was performed using SPSS version 13.0 (SPPS, Inc., Chicago, IL, USA). Continuous variables were expressed as the mean \pm standard error and analyzed using the Student's $t$-test (two-tailed), $U$ test, $\chi^{2}$ test and Fisher's exact test. Spearman's rank correlation analysis was performed to determine the correlation between two rank-order variables. $\mathrm{P}<0.05$ was considered to indicate a statistically significant difference.

\section{Results}

RKIP expression levels are reduced in NSCLC cancerous tissues. Following obtaining ethical approval and informed patient consent, 100 patients with NSCLC were recruited to the present study. The clinicopathological information of patients is summarized Table I. There were 21 cases of TNM stage I/II and 79 cases of stage III/IV NSCLC. The variation in levels of RKIP protein expression between these two groups was statistically significant $(\mathrm{P}=0.008)$, with reduced RKIP expression levels in patients with late-stage NSCLC. A total of 82 patients had lymph node metastasis, whereas 18 were negative for metastasis. Of these, $47(57.32 \%)$ patients with metastasis exhibited low RKIP expression levels and 9 (50.0\%) of the patients without metastasis had high RKIP expression levels, a difference that was statistically significant $(\mathrm{P}=0.001)$. When NSCLC distant metastasis was compared, 63 patients (65.08\%) had developed distant metastasis and exhibited lower levels of RKIP expression, whereas 27 patients $(72.97 \%)$ had intra-lung metastasis with significantly higher levels of RKIP expression $(\mathrm{P}=0.018)$.

Histological sections of cancer and non-cancer tissues were stained with anti-RKIP or anti-pSTAT3 antibodies. Negative or weak staining of RKIP was observed in the majority of cancerous tissues compared with intense staining in non-cancerous tissues (Fig. 1A). However, intense pSTAT3 staining was observed in cancerous tissues (Fig. 1A). Bar chart quantification of RKIP and pSTAT3 positive staining revealed a significant difference between cancerous and non-cancerous tissues (Fig. 1B; $\mathrm{P}=0.00256$; paired $t$-test). Furthermore, patients with long distance metastasis exhibited lower RKIP expression levels compared with patients with within-lung metastasis ( $\mathrm{P}=0.018$; Table $\mathrm{I})$.

Reduced RKIP expression levels are associated with NSCLC intra-lung or long distance metastasis. Tissues obtained from patients that were lymph node metastasis-positive and -negative were examined using western blotting. The results revealed that patients that were positive for lymph node metastasis had lower levels of RKIP protein expression, as compared with tissues from patients that were negative for metastasis (Fig. 2A). The grayscale density of each band was evaluated using Photoshop software, and calculated in Prism 5.0 software. The results revealed that tissue from patients with lymph node metastasis had significantly lower levels of RKIP expression compared with that of patients without metastasis (Fig. 2B; $\mathrm{P}=0.0013 ; \chi^{2}$ test). This finding indicated that NSCLC metastasis may be associated with RKIP expression.

RKIP blocks IL-6-induced phosphorylation-mediated STAT3 activation and inhibits NSCLC cells metastasis. In order to elucidate whether RKIP overexpression is able to inhibit STAT3 phosphorylation in NSCLC cells, the RKIP gene was constructed into pLenti6.3/V5-DEST lentiviral vectors. Initially, the infective efficiency of the constructed RKIP lentivirus vector was assessed by examining GFP fluorescence, indicating that $\geq 90 \%$ of the cancer cells were infected with viral vectors (Fig. 3A), and western blot analysis also demonstrated RKIP overexpression (data not shown). The western blotting results revealed that overexpression of RKIP significantly suppressed IL-6-dependent ERK and STAT3 phosphorylation (Fig. 3B, $\mathrm{P}=0.028$ ). Upon overexpression of RKIP through Lenti7.3-RKIP infection, the Transwell invasive ability of NSCLC cells decreased (Fig. 3C, P=0.034). Therefore, the results demonstrated that RKIP overexpression was able to inhibit STAT3 phosphorylation and cell migration in NSCLC cell lines. 
Table I. Clinicopathological characteristics of patients with NSCLC and RKIP expression levels.

\begin{tabular}{|c|c|c|c|c|c|}
\hline \multirow[b]{2}{*}{ Clinical characteristics } & \multirow[b]{2}{*}{ Number of cases } & \multicolumn{3}{|c|}{ RKIP expression levels } & \multirow[b]{2}{*}{ P-value } \\
\hline & & Low $(\%)$ & Moderate (\%) & High $(\%)$ & \\
\hline \multicolumn{6}{|l|}{ Gender } \\
\hline Male & 76 & $40(52.63)$ & $23(30.26)$ & $13(17.11)$ & 0.341 \\
\hline Female & 24 & $9(37.50)$ & $11(45.83)$ & $4(16.67)$ & \\
\hline \multicolumn{6}{|l|}{ Age } \\
\hline$\geq 60$ & 56 & $18(32.14)$ & $23(41.07)$ & 15 (26.79) & 0.452 \\
\hline$<60$ & 44 & $19(43.18)$ & $13(29.55)$ & $12(27.27)$ & \\
\hline \multicolumn{6}{|l|}{ TNM stage } \\
\hline $\mathrm{I} / \mathrm{II}$ & 21 & $12(57.14)$ & $7(33.33)$ & $2(9.52)$ & 0.008 \\
\hline III/IV & 79 & $41(51.90)$ & $23(29.11)$ & 15 (18.99) & \\
\hline \multicolumn{6}{|l|}{ Lymph node metastasis } \\
\hline Positive & 82 & $47(57.32)$ & $22(26.83)$ & $13(15.85)$ & 0.001 \\
\hline Negative & 18 & $5(27.78)$ & $4(22.22)$ & $9(50.00)$ & \\
\hline \multicolumn{6}{|l|}{ Distant metastasis } \\
\hline Within-lung & 37 & $3(8.11)$ & 7 (18.92) & $27(72.97)$ & 0.018 \\
\hline Long distance & 63 & $41(65.08)$ & $17(26.98)$ & $5(7.94)$ & \\
\hline
\end{tabular}

RKIP, Raf kinase inhibitor protein; NSCLC, non-small cell lung cancer; TNM, tumor node metastasis.
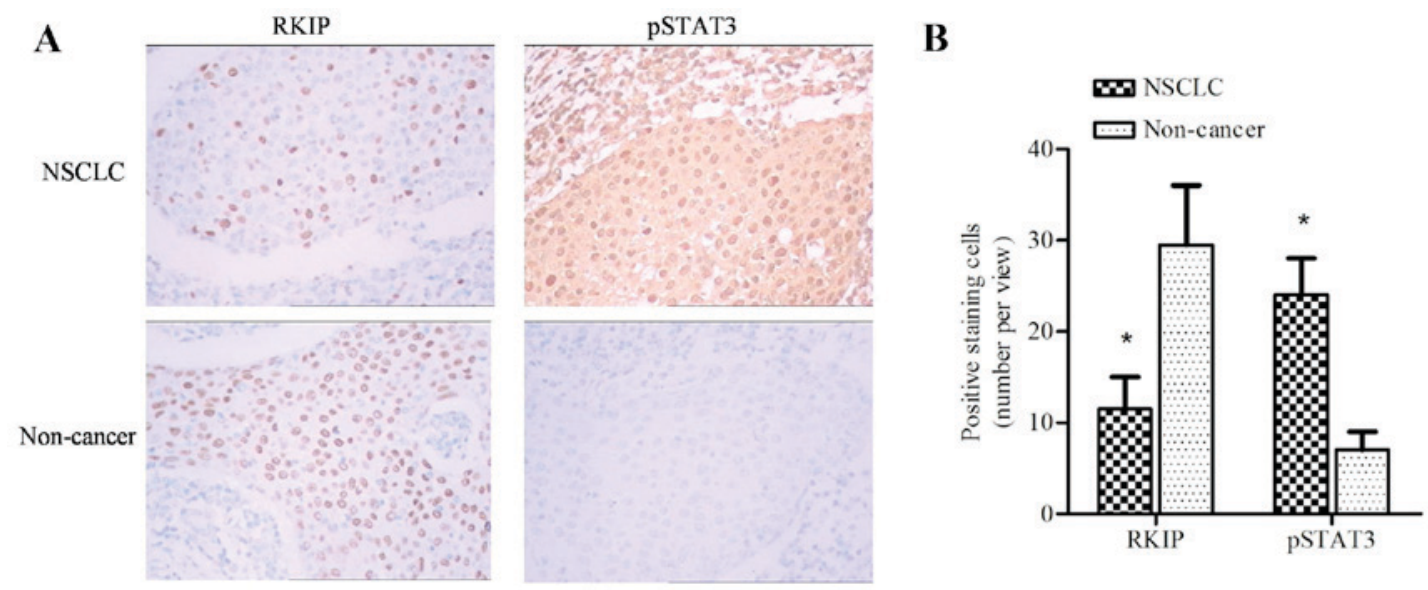

Figure 1. (A) Paired cancer and non-cancer tissues from patients with NSCLC were stained with anti-RKIP or anti-pSTAT3 antibody. (B) Counting of RKIP and pSTAT3-positively stained cells under phase-contrast microscopy are presented in bar charts produced using Prism 5.0 software $\left({ }^{*} \mathrm{P}<0.05\right.$; paired $t$-test; error bars represent the mean + standard deviation). NSCLC, non-small cell lung cancer; pSTAT3, phosphorylated signal transducer and activator of transcription 3; RKIP, Raf kinase inhibitor protein.

RKIP knockdown promotes STAT3 phosphorylation and NSCLC-cell metastasis. A549 and NCI-H1299 NSCLC cells were seeded into 6 -well plates $24 \mathrm{~h}$ prior to RKIP siRNA interference. Transfection reagent-mediated siRNA interference was administered to cancer cells. At $48 \mathrm{~h}$ following incubation, cells were collected and analyzed. Western blot results revealed that STAT3 phosphorylation was decreased in the presence of IL- 6 stimulation in the two transfected cell lines, compared with the control group (Fig. 4A and B).

RKIP overexpression suppressed xenograft tumor metastasis in nude mice. The in vivo study was designed to mimic the progressive growth of lung metastatic tumors through tail vein injection of infected or control A549 NSCLC cells at a density of $1 \times 10^{6}$ cells/mouse. At 6 weeks following transplantation, bioluminescence was utilized to conduct the lung cancer cell-derived tissue metastasis assay. Fluorescence signals from mice were detected using the Xenogen IVIS imaging system. The results indicated that the RKIP overexpression group had lower numbers of metastatic tumors within the lung or the whole body, compared with the control group (Fig. 5A; $\mathrm{P}=0.0063$; Fisher's exact test). Hematoxylin and eosin staining was performed on mouse tissues, revealing that the lungs exhibited reduced tumor mass in the RKIP overexpression group, compared with the control group (Fig. 5B). The experimental results indicated the overexpression of 


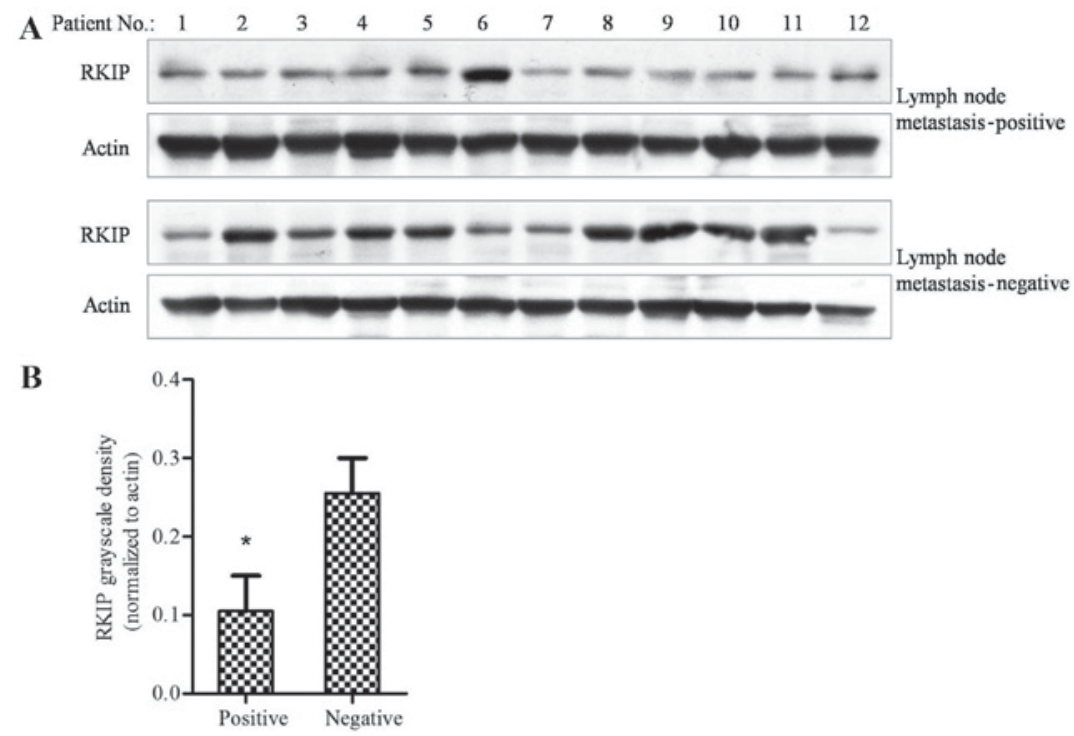

Figure 2. (A) Western blot analysis of RKIP expression levels in tissue from lymph node metastasis-positive or lymph node metastasis-negative patients with NSCLC. (B) The grayscale density of RKIP protein expression was evaluated using Photoshop software. The comparison of RKIP expression levels between these two sub-groups was analyzed using the Mann-Whitney U-test ("P $<0.05$; error bars represent the mean + standard deviation). NSCLC, non-small cell lung cancer; RKIP, Raf kinase inhibitor protein.
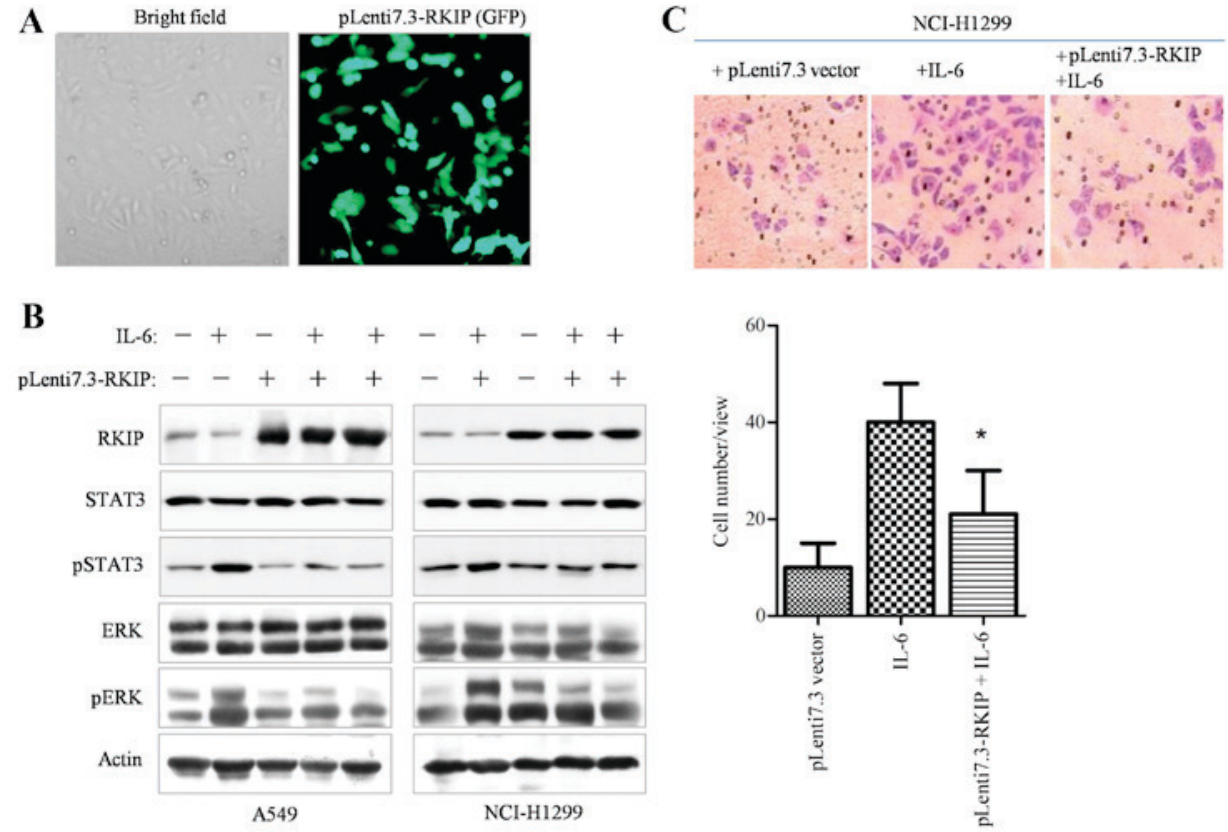

Figure 3. (A) GFP detection indicated that $\geq 90 \%$ of NSCLC cells were effectively infected with virus particles. (B) Western blotting results revealed that overexpressed RKIP significantly suppressed ERK and STAT3 phosphorylation in A549 and NCI-H1299 cells. (C) Transwell invasion assay was performed to assess the invasiveness of NSCLC cells infected with blank lentivirus or RKIP-containing lentivirus. Representative images of invaded cells are shown, and 5 random fields were counted per well ("P<0.05). GFP, green fluorescent protein; NSCLC, non-small cell lung cancer; ERK, extracellular signal-regulated kinase; pERK, phosphorylated extracellular signal-regulated kinase; STAT3, signal transducer and activator of transcription 3; pSTAT3, phosphorylated signal transducer and activator of transcription 3; IL-6, interleukin 6; RKIP, Raf kinase inhibitor protein.

RKIP may block A549 cancer cell metastasis and growth through the blockade of downstream signaling pathways, including the RAF/ERK/STAT3 pathway.

\section{Discussion}

The poor prognosis and shorter survival time of patients with NSCLC are closely associated with tumor cell aggressive and long distance metastasis (2). The biological behavior of NSCLC cells may also impact the efficacy of therapeutic agents, due to its malignancy $(2,20)$. In the present study, based on the data from 100 patients with NSCLC, negative or weak staining of RKIP was observed in the majority of cancerous tissues, compared with an intense signal in non-cancerous tissues (Fig. 1A). Patients at an increased clinical stage (III/IV) exhibited lower levels of RKIP expression, 
A

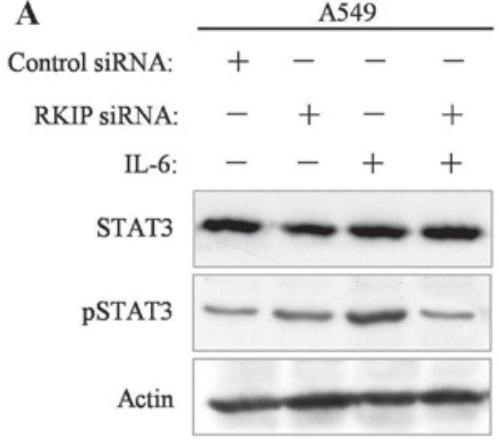

B

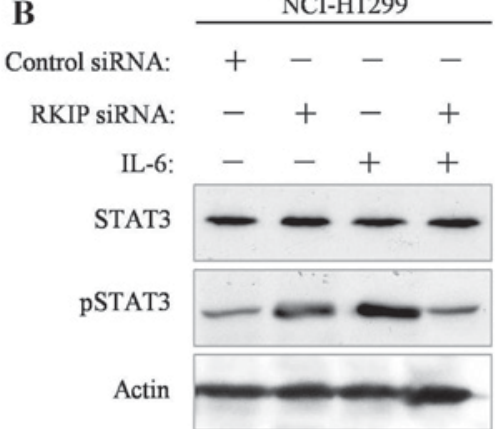

Figure 4. Western blot analysis revealed that siRNA-mediated RKIP silencing blocked IL-6-induced STAT3 phosphorylation in (A) A549 cells and (B) NCI-H1299 cells. RKIP, Raf kinase inhibitor protein; IL-6, interleukin 6; STAT3, signal transducer and activator of transcription; pSTAT3, phosphorylated signal transducer and activator of transcription 3; siRNA, small interfering RNA.

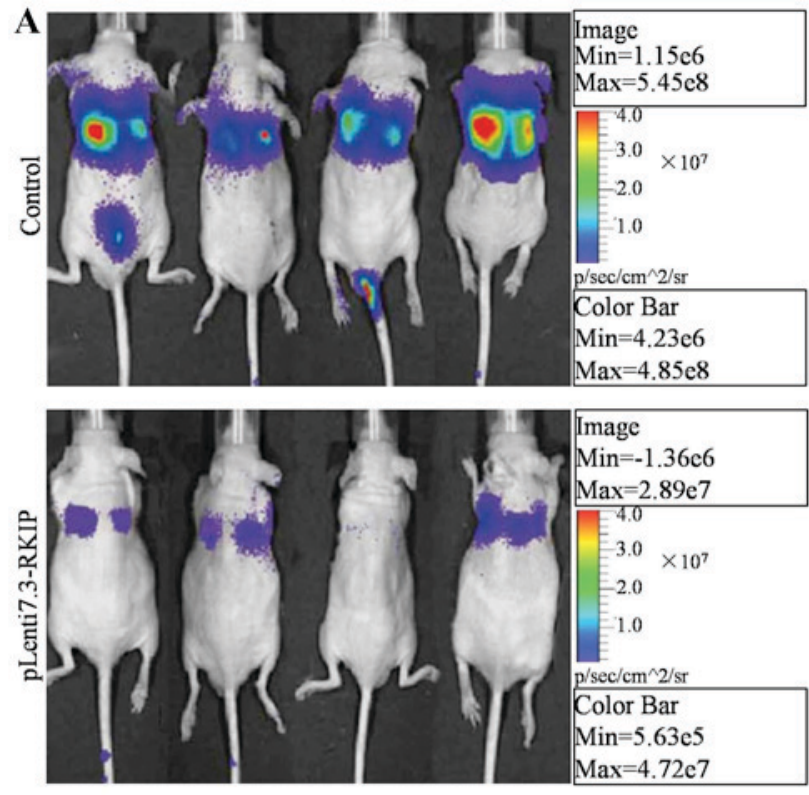

B
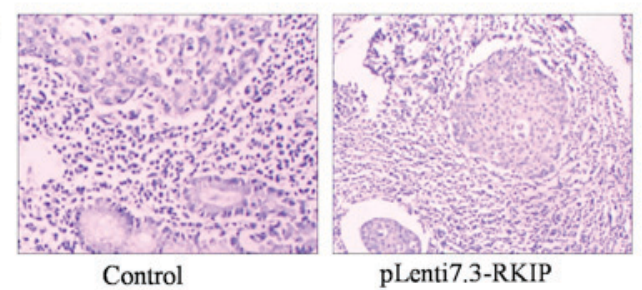

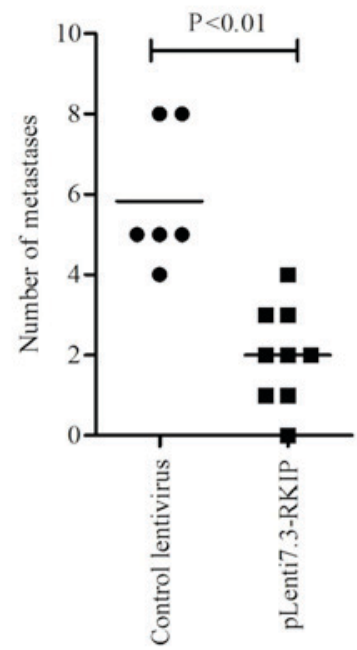

Figure 5. (A) Xenogen IVIS imaging of xenograft-bearing mice, which were injected with normal A549 cells or RKIP-overexpressing A549 cells through the tail vein. Counting of metastasis hotspots from these two groups is presented in the bar chart. (B) Hematoxylin and eosin staining was performed on tumor tissues. RKIP, Raf kinase inhibitor protein.

whereas stage I/II patients had higher levels of RKIP expression and an improved prognosis. In total, $57.32 \%$ patients with lymph node metastasis exhibited low levels of RKIP expression, but $50 \%$ of lymph node metastasis-negative patients had high levels of RKIP expression. Additionally, the statistical data revealed that $65 \%$ of long-distance metastasis patients had low levels of RKIP expression, whereas $>72 \%$ of patients with within-lung metastasis exhibited high levels of RKIP expression ( $\mathrm{P}=0.018$, Table I). Taken together, these findings reveal that NSCLC metastasis is inversely correlated with RKIP expression levels. This result is concordant with a previous study examining NSCLC and RKIP expression (12).
A previous study revealed that silenced Raf expression promoted tumor invasive ability, whereas its upregulation suppressed tumor invasive ability (21). Other previous studies have indicated that Raf kinase activated transcriptional activity via activation of the ERK signaling pathway $(22,23)$. RKIP is a negative regulator of the RAS-mitogen-activated protein kinase and ERK signaling pathway (24). A previous study indicated using immunohistochemistry that RKIP expression levels were detectable in all non-cancerous prostate tissues and were decreased to low or undetectable levels in all prostate cancer metastases (25). Similar results have been demonstrated in malignant melanoma, colorectal, breast, thyroid and liver 
cancer (26-28). Concordant with the results from previous studies, the current study also revealed that RKIP may be associated with the Raf/MEK/ERK/STAT3 pathway, and clinical data from 100 patients with NSCLC also supported the hypothesis that RKIP expression levels are associated with NSCLC metastasis. Patients with positive metastatic lymph nodes, with intra-lung or long-distance metastasis, had lower RKIP protein levels (Figs. 1 and 2; Table I), and lower RKIP expression levels were observed with increasing TNM stage. Previous findings have demonstrated that pRKIP is a predictive indicator of lung cancer survival (24). Therefore, future studies may further examine the role of RKIP in NSCLC, as well as investigating pRKIP, its kinase and underlying mechanisms in this form of cancer.

In conclusion, the present study suggests an important role for RKIP in NSCLC on the basis of in vitro data, in vivo metastatic experiments and clinical statistic analysis. The findings strengthen the hypothesis that RKIP suppresses NSCLC cell metastasis through blocking of the Raf/ERK/STAT3 signaling pathway.

\section{Acknowledgements}

The current study was supported by the Natural Science Foundation of Anhui Province (grant no. 1408085MH144).

\section{References}

1. Siegel RL, Miller KD and Jemal A: Cancer statistics, 2015. CA Cancer J Clin 65: 5-29, 2015

2. Black RC and Khurshid H: NSCLC: An update of driver mutations, their role in pathogenesis and clinical significance. R I Med J (2013) 98: 25-28, 2015.

3. Alberg AJ, Brock MV, Ford JG, Samet JM and Spivack SD: Epidemiology of lung cancer: Diagnosis and management of lung cancer, 3rd ed: American College of Chest Physicians evidence-based clinical practice guidelines. Chest 143 (5 Suppl): e1S-e29S, 2013.

4. Tang Z, Li J, Shen Q, Feng J, Liu H, Wang W, Xu L, Shi G, Ye X, Ge M, et al: Contribution of upregulated dipeptidyl peptidase 9 (DPP9) in promoting tumoregenicity, metastasis and the prediction of poor prognosis in non-small cell lung cancer (NSCLC). Int J Cancer: Dec 10, 2016 (Epub ahead of print).

5. Goyette MA and Côté JF: NSCLC metastasis: Going with ELMO3. Oncotarget 5: 5850-5851, 2014.

6. Escara-Wilke J, Yeung K and Keller ET: Raf kinase inhibitor protein (RKIP) in cancer. Cancer Metastasis Rev 31: 615-620, 2012.

7. Deiss K, Kisker C, Lohse MJ and Lorenz K: Raf kinase inhibitor protein (RKIP) dimer formation controls its target switch from Raf1 to G protein-coupled receptor kinase (GRK) 2. J Biol Chem 287: 23407-23417, 2012.

8. Farooqi AA, Li Y and Sarkar FH: The biological complexity of RKIP signaling in human cancers. Exp Mol Med 47: e185, 2015.

9. Yun J, Frankenberger CA, Kuo WL, Boelens MC, Eves EM, Cheng N, Liang H, Li WH, Ishwaran H, Minn AJ and Rosner MR: Signalling pathway for RKIP and Let-7 regulates and predicts metastatic breast cancer. EMBO J 30: 4500-4514, 2011.

10. Jia B, Liu H, Kong Q and Li B: RKIP expression associated with gastric cancer cell invasion and metastasis. Tumour Biol 33: 919-925, 2012.

11. Fu Z, Kitagawa Y, Shen R, Shah R, Mehra R, Rhodes D, Keller PJ, Mizokami A, Dunn R, Chinnaiyan AM, et al: Metastasis suppressor gene Raf kinase inhibitor protein (RKIP) is a novel prognostic marker in prostate cancer. Prostate 66: 248-256, 2006
12. Wang Q, Wu X, Wu T, Li GM and Shi Y: Clinical significance of RKIP mRNA expression in non-small cell lung cancer. Tumour Biol 35: 4377-4380, 2014.

13. Tan FH, Putoczki TL, Stylli SS and Luwor RB: The role of STAT3 signaling in mediating tumor resistance to cancer therapy. Curr Drug Targets 15: 1341-1353, 2014.

14. Saxena NK, Sharma D, Ding X, Lin S, Marra F, Merlin D and Anania FA: Concomitant activation of the JAK/STAT, PI3K/AKT, and ERK signaling is involved in leptin-mediated promotion of invasion and migration of hepatocellular carcinoma cells. Cancer Res 67: 2497-2507, 2007.

15. Jin HO, Lee YH, Park JA, Kim JH, Hong SE, Kim HA, Kim EK, Noh WC, Kim BH, Ye SK, et al: Blockage of Stat3 enhances the sensitivity of NSCLC cells to PI3K/mTOR inhibition. Biochem Biophys Res Commun 444: 502-508, 2014.

16. Gorgisen G, Ozes D, Pehlivanoglu S, Erdogan A, Dertsiz L, Ozbilim G, Ozbudak IH, Savas B and Ozes ON: Differential expression and activation of epidermal growth factor receptor 1 (EGFR1), ERK, AKT, STAT3, and TWIST1 in nonsmall cell lung cancer (NSCLC). Exp Lung Res 39: 387-398, 2013.

17. Ye CG, Chen GG, Ho RL, Merchant JL, He ML and Lai PB: Epigenetic upregulation of Bak by ZBP-89 inhibits the growth of hepatocellular carcinoma. Biochim Biophys Acta 1833: 2970-2979, 2013

18. Zhang G, Liu F, Chen XY, Fang WY, Li HL, Huang ZP, Chu HJ, Wang $\mathrm{X}$ and Zhao T: Construction and identification of a recombinant lentivirus harboring small interfering RNA targeting mouse CD99 antigen-like 2 gene. Nan Fang Yi Ke Da Xue Xue Bao 29: 228-231, 2009 (In Chinese)

19. Zheng J, Wang J, Sun X, Hao M, Ding T, Xiong D, Wang X, Zhu Y, Xiao G, Cheng G, et al: HIC1 modulates prostate cancer progression by epigenetic modification. Clin Cancer Res 19: 1400-1410, 2013.

20. Reinmuth N, Stumpf P, Stumpf A, Muley T, Kobinger S, Hoffmann H, Herth FJ, Schnabel PA, Bischoff $\mathrm{H}$ and Thomas M: Characteristics of lung cancer after a previous malignancy. Respir Med 108: 910-917, 2014

21. Chatterjee D, Sabo E, Tavares R and Resnick MB: Inverse association between Raf kinase inhibitory protein and signal transducers and activators of transcription 3 expression in gastric adenocarcinoma patients: Implications for clinical outcome. Clin Cancer Res 14: 2994-3001, 2008.

22. Ulivi P, Arienti C, Amadori D, Fabbri F, Carloni S, Tesei A, Vannini I, Silvestrini R and Zoli W: Role of RAF/MEK/ERK pathway, p-STAT-3 and Mcl-1 in sorafenib activity in human pancreatic cancer cell lines. J Cell Physiol 220: 214-221, 2009.

23. Aziz MH, Hafeez BB, Sand JM, Pierce DB, Aziz SW, Dreckschmidt NE and Verma AK: Protein kinase Cvarepsilon mediates Stat3Ser727 phosphorylation, Stat3-regulated gene expression, and cell invasion in various human cancer cell lines through integration with MAPK cascade (RAF-1, MEK1/2, and ERK1/2). Oncogene 29: 3100-3139, 2010.

24. Huerta-Yepez S, Yoon NK, Hernandez-Cueto A, Mah V, Rivera-Pazos CM, Chatterjee D, Vega MI, Maresh EL, Horvath S, Chia D, et al: Expression of phosphorylated raf kinase inhibitor protein (pRKIP) is a predictor of lung cancer survival. BMC Cancer 11: 259, 2011.

25. Yousuf S, Duan M, Moen EL, Cross-Knorr S, Brilliant K, Bonavida B, LaValle T, Yeung KC, Al-Mulla F, Chin E and Chatterjee D: Raf kinase inhibitor protein (RKIP) blocks signal transducer and activator of transcription 3 (STAT3) activation in breast and prostate cancer. PLoS One 9: e92478, 2014.

26. Cross-Knorr S, Lu S, Perez K, Guevara S, Brilliant K, Pisano C, Quesenberry PJ, Resnick MB and Chatterjee D: RKIP phosphorylation and STAT3 activation is inhibited by oxaliplatin and camptothecin and are associated with poor prognosis in stage II colon cancer patients. BMC Cancer 13: 463, 2013.

27. Datar I, Qiu X, Ma HZ, Yeung M, Aras S, de la Serna I, Al-Mulla F, Thiery JP, Trumbly R, Fan X, et al: RKIP regulates CCL5 expression to inhibit breast cancer invasion and metastasis by controlling macrophage infiltration. Oncotarget 6: 39050-39061, 2015.

28. Zhang XM, Gu H, Yan L and Zhang GY: RKIP inhibits the malignant phenotypes of gastric cancer cells. Neoplasma 60: 196-202, 2013. 